\title{
Le statut linguistique des pronoms du créole haïtien et du français : une étude de corpus ${ }^{1}$
}

\author{
Isabelle Barriere ${ }^{1}$, Géraldine Legendre ${ }^{2}$, Blandine Joseph ${ }^{2}$, Sarah Kresh $^{3}$, Prince Fleurio Guetjen ${ }^{4}$ et Thierry Nazzi ${ }^{5}$ \\ ${ }^{1}$ Long Island University/Brooklyn and Yeled V'Yalda (États-Unis), \\ 2 Johns Hopkins University (États-Unis), \\ ${ }^{3}$ City University of New York Graduate Center (États-Unis), \\ ${ }^{4}$ Ecole Nationale des Arts (Haïti), \\ ${ }^{5}$ Laboratoire Psychologie de la Perception (LPP) - UMR 8242 CNRS- Université Paris-Descartes (France) \\ isabelle.barriere@gmail.com, legendre@jhu.edu, blandinej1@gmail.com, sarahkresh@yahoo.ca, \\ princeboiscaiman@yahoo.fr, thierry.nazzi@parisdescartes.fr
}

\begin{abstract}
Résumé. Le statut syntaxique et phonologique des pronoms en créole haïtien et en français a fait l'objet de débats. Les analyses linguistiques classiques du français formulées dans la tradition générative ont proposé une distinction entre les pronoms forts et les pronoms faibles ou 'clitiques'. Tandis que les pronoms forts se comportent comme des syntagmes nominaux (SN), les clitiques diffèrent de ceux-ci de par leur distribution syntaxique. Ces premières analyses se fondent sur des jugements de grammaticalité et certaines de ces analyses ont été remises en question lorsque des corpus de parole spontanée ont été mis a la disposition des chercheurs et ont permis des analyses quantitatives et phonologiques. La présente étude comparative a pour objectif l'analyse des pronoms personnels du français et du créole haïtien et se fondent sur l'analyse de corpus CHILDES (Demuth \& Tremblay, 2008; MacWhinney, 2000) pour le français et un nouveau corpus que nous avons enregistré et transcrit pour le créole haïtien. Les analyses portent sur la distribution syntaxique des pronoms sujets et objets et sur leurs réductions phonologiques. Ces deux sources d'information nous permettent de conclure aux statuts contrastifs des pronoms en créole haïtien qui arborent toutes les caractéristiques des pronoms forts en ce qui concerne leur distribution syntaxique tandis qu'au niveau phonologique ils peuvent être réduits et se comportent donc comme des clitiques phonologiques. En revanche le français comprend des pronoms forts et des pronoms faibles/clitiques qui se distinguent de part leurs trait phonologiques et leur distribution syntaxique.
\end{abstract}

\begin{abstract}
The syntactic and phonological status of pronouns in Haitian Creole and French has been debated. The linguistic analyses formulated in the generative framework made a distinction between strong and weak pronouns or 'pronominal clitics'. While strong pronouns behave like Determiner Phrases (SN), clitics differ with respect their syntactic distribution. These early analyses were based on judgments of grammaticality and a few of these were questioned when spontaneous speech corpora were made available and gave rise to quantitative and phonological analysis. The present comparative compares the personal pronouns of French and Haitian Creole. It is based the analyses of corpora- CHILDES (Demuth \& Tremblay, 2008; MacWhinney, 2000) for the French and a new body that we compiled and transcribed for Haitian Creole. The analyses focus on the syntactic distribution of subjects and objects pronouns and their phonological realizations. On the basis of these analyse, we conclude that Haitian Creole bear all the characteristics of strong pronouns regarding their syntactic distribution while at the phonological level can be reduced and behave like clitics. In contrasct, French pronouns include strong and weak pronouns / clitic pronouns that differ in their phonological realizations and their syntactic distribution.
\end{abstract}


Le statut des pronoms en créole haïtien et en français a fait l'objet de débats. Les analyses linguistiques classiques (e.g. Kayne, 1977; Ruwet, 1976) du français formulées dans la tradition générative ont proposé une distinction entre les pronoms forts et les pronoms faibles ou 'clitiques'. Tandis que les pronoms forts se comportent clairement comme des syntagmes nominaux (SN), les clitiques diffèrent de ceux-ci de par leur propriétés distributionnelles syntaxiques et phonologiques. Ces premières analyses se fondent sur des jugements de grammaticalité et certaines de ces analyses ont été remises en question lorsque des corpus de parole spontanée ont été mis a la disposition des chercheurs et ont permis des analyses quantitatives et phonologiques. Pour le créole haïtien il n'existe que quelques études syntaxiques préalables basées sur des jugements de grammaticalité et leurs conclusions sont radicalement opposées (e.g. De Graff, 1993 vs. Déprez, 1994).

La présente étude comparative a pour objectif l'analyse des pronoms Personnels du français et du créole haïtien et se fondent sur l'analyse de corpus CHILDES (Demuth \& Tremblay, 2008; MacWhinney, 2000) pour le français et un nouveau corpus que nous avons enregistré et transcrit pour le créole haïtien. Les analyses portent sur la distribution syntaxique des pronoms sujets et objets et sur leurs réductions phonologiques. Ces deux sources d'information nous permettent de conclure concernant le statut contrastif des pronoms en créole haïtien qui arborent toutes les caractéristiques des pronoms forts en ce qui concerne leur distribution syntaxique tandis qu'au niveau phonologique ils peuvent être réduits et se comportent donc comme des clitiques phonologiques. En revanche le français comprend des pronoms forts et des pronoms faibles/clitiques qui se distinguent de part leurs trait phonologiques et leur distribution syntaxique.

\section{Introduction}

Dans les études linguistiques des langues romanes qui s’inscrivent dans des cadres linguistiques différents, de nombreux auteurs distinguent les pronoms faibles ou clitiques d'un côté et les pronoms forts et syntagmes nominaux, de l'autre (Kayne, 1975, 1977 ; Ruwet, 1976 ; Blanche-Benveniste et al., 1987 ; Zribi-Hertz, 1997). Le terme clitique est utilisé de facon ambigüe dans la littérature afin d'indiquer des éléments linguistiques qu'il est souvent difficile de catégoriser. D’une certaine façon, ils se comportent comme des mots indépendants ou morphèmes libres. Simultanément ils arborent des traits morphosyntaxiques qui caractérisent les morphèmes liés (pour une longue discussion, voir Klavans, 1980). Les clitiques sont souvent sujets à des réductions phonologiques qui donnent lieu à des contractions et des phénomènes de restructuration syllabique comme la liaison ou l'élision. Dans le contexte de l'analyse syntaxique des langues romanes, le terme pronom faible ou clitique pronominal s'applique à une catégorie de pronoms qui ne se comportent pas comme des SN et qui ont des pronoms forts qui leur correspondent. Certains traits qui définissent les clitiques français ont été identifiés. D’après Kayne $(1975,1977)$ qui est parmi les premiers générativistes à discuter des propriétés du système pronominal en français, les traits distinctifs des clitiques pronominaux comprennent la liste ci-dessous :

a) Il ne peuvent pas porter la marque de l'accent tonique contrastif, ce qui explique l'agrammaticalité de $1 \mathrm{a}$ et $1 \mathrm{~b}^{2}$.

(1) a *IL sort.

$\mathrm{b} *$ Je LA vois.

b) Ils n’apparaissent pas indépendamment d'autres éléments dans une phrase, ce qui rend compte de l'agrammaticalité de 2a et 3a contrastant avec la grammaticalité de $2 b$ et 3b, qui contiennent des pronoms forts et des syntagmes lexicaux.

(2) a Qui est arrivé? *il

b Qui est arrivé? Lui/le facteur

(3) a Qui as-tu rencontré? *Le

b Qui as-tu rencontré? Lui/le facteur 
c) Ils ne peuvent pas être conjoints, ce qui explique l'agrammaticalité de 4a et 5a, qui s'oppose à la grammaticalité de $4 b$, 4c et $5 b$.

(4) a * Il et elle prennent le train

b Lui et elle ${ }^{3}$ (ils) prennent le train

c Jean et Marie prennent le train

(5) a * Je le et la regarde

b Je regarde Jean et Marie

d) Ils ne peuvent pas apparaître dans les constructions négatives restrictives, ce qui rend compte de l'agrammaticalité de 6a qui s’oppose à la grammaticalité de 6b qui contient un pronom fort et 6c qui contient un SN.

(6) a *Catherine n'aime que les

b Catherine n'aime qu'eux

c Catherine n'aime que les chiens

Les clitiques sont aussi sélectifs quant aux éléments qui peuvent les séparer de leur hôte, c’est-à-dire dans le cas des clitiques sujets et objets des verbes auxquels ils se cliticisent. Par exemple les clitiques objets ne peuvent être séparés de leur hôte que par un autre clitique, ou les marques de négation, autrement dit des catégories fonctionnelles, ce qui explique la grammaticalité des énoncés 7 et 8a extraites du corpus de Lyon (Demuth \& Tremblay, 2008) et l'agrammaticalité de l'exemple 8b.

(7) Où tu l'as mis le biberon ? (Ana3, ligne 710)

(8) a Tu as tout démonté hein (Ana2, ligne 167)

b *Tu tout as démonté

Deux autres caractéristiques s’appliquent aux clitiques sujets et objets en français. Les clitiques objets n'occupent pas la même position que les arguments (nominaux et pronominaux forts) auxquels ils se référent puisqu'ils sont préverbaux. En ce qui concerne les sujets clitiques, en particulier dans la parole spontanée courante du français moderne, ils ont le statut de marques d’accord qui seraient générés au niveau syntaxique dans les têtes fonctionnelles (e.g. Zribi-Hertz, 1997; Culbertson et Legendre, 2008 ; Culbertson, 2010). Dans le registre soutenu du français moderne leur statut est moins clair car ce registrelà présente les caractéristiques d'un processus diachronique non-achevé. Ce processus diachronique d'évolution d'un pronom argument vers une marque d'accord est un processus attesté dans beaucoup de langues (Corbett, 2006 ; Fuß, 2005 ; Hopper \& Traugott, 1993) ; en français il semblerait qu'il ne soit achevé que dans le registre oral spontané de la langue (ou écrit du type SMS ou Chat sur internet).

Le statut des pronoms dans les 'créoles' dits 'français' a aussi fait l'objet de plusieurs analyses (e.g. Baptista, 1995) y compris le créole haïtien. Les tableaux 1 et 2 ci-dessous présentent les paradigmes des pronoms Personnels et possessifs et déterminants possessifs en français (tableau 1) et en créole haïtien (tableau 2). L'organisation du tableau 1 indique que les formes des clitiques sujets du français se distinguent des pronouns forts pour de nombreuses Personnes (première, deuxième Personne du singulier ; troisième Personne du singulier au masculin, et troisième Personne du pluiel). Ces distinctions de forme s'appliquent aussi à la distinction entre pronoms objets et clitiques objets. En revanche, les réductions phonologiques des pronoms haïtiens constituent des contractions des pronoms forts et sont donc directement dérivées de ces pronoms forts. 
$\underline{\text { Tableau } 1 \text { : Paradigme des pronoms Personnels et possessifs et déterminants possessifs en français }}$

\begin{tabular}{|c|c|c|c|c|c|c|c|c|c|}
\hline \multirow[t]{2}{*}{ Nombre } & \multirow[t]{2}{*}{ Personne } & \multicolumn{2}{|l|}{ Sujet } & \multicolumn{2}{|c|}{ Objet Direct } & \multirow{2}{*}{$\begin{array}{l}\text { Objet } \\
\text { Indirect } \\
\text { IOCL } \\
\end{array}$} & \multirow{2}{*}{$\begin{array}{l}\text { 'Réfléchis' } \\
\text { SECL } \\
\end{array}$} & \multirow{2}{*}{$\begin{array}{l}\text { Pronom } \\
\text { Possessifs } \\
\text { POSPn } \\
\end{array}$} & \multirow{2}{*}{$\begin{array}{l}\text { Déterminants } \\
\text { Possessifs } \\
\text { POSDet } \\
\end{array}$} \\
\hline & & SPn* & SCL & DOPn & DOCL & & & & \\
\hline \multirow[t]{3}{*}{ SG } & $1^{\text {ère }}$ & moi & je/j' & moi & me & me & me & mien(ne)(s) & mon/ma \\
\hline & $2^{\text {ème }}$ & $\begin{array}{l}\text { toi } \\
\text { vous }\end{array}$ & $\begin{array}{l}\text { tu/t' } \\
\text { vous }\end{array}$ & $\begin{array}{l}\text { toi } \\
\text { vous }\end{array}$ & $\begin{array}{l}\text { te } \\
\text { vous }\end{array}$ & $\begin{array}{l}\text { te } \\
\text { vous }\end{array}$ & te & $\begin{array}{l}\text { tien(ne)(s) } \\
\text { votre(s) }\end{array}$ & $\begin{array}{l}\text { ton/ta } \\
\text { votre }\end{array}$ \\
\hline & $3^{\text {ème }}$ & $\begin{array}{l}\text { lui } \\
\text { elle }\end{array}$ & $\begin{array}{l}\text { il } \\
\text { elle }\end{array}$ & $\begin{array}{l}\text { lui } \\
\text { elle }\end{array}$ & $\begin{array}{l}\text { le } \\
\text { la } \\
\end{array}$ & lui & se & sien(ne)(s) & son/sa \\
\hline \multirow[t]{3}{*}{ PL } & $1^{\text {ère }}$ & nous & $\begin{array}{l}\begin{array}{l}\text { nous } \\
\text { on }\end{array} \\
\end{array}$ & nous & nous & nous & nous & notre(s) & notre/nos \\
\hline & $2^{\text {ème }}$ & vous & vous & vous & vous & vous & vous & votre(s) & votre/vos \\
\hline & $3^{\text {ème }}$ & $\begin{array}{l}\text { eux } \\
\text { elles }\end{array}$ & $\begin{array}{l}\text { ils } \\
\text { elles }\end{array}$ & $\begin{array}{l}\text { eux } \\
\text { elles }\end{array}$ & les & leur & se & leur(s) & \\
\hline
\end{tabular}

${ }^{*} \mathrm{SPn}=$ Pronom Sujet ; SCL= Clitique Sujet ; DOPn= Pronom Objet Direct ; DOCL= Clitique Objet Direct ; IOCL= DOCL= Clitique Objet Indirect ; SECL= Clitique SE dit 'Réfléchi' (à valeur Réciproque, Anti-causative, Intrinsèque, Médio-passive, de Datif Ethique et qui entre en jeu dans l'expression de la Possession Inaliénable); POSPn= Pronom Possessif ; POSTDet= Déterminants possessifs

Les pronoms sujets du créole haïtien ont donné naissance à deux positions théoriques opposées. D’après DeGraff (1993) il s'agirait de marques d'accord (ou 'clitiques syntaxiques' ou 'pronoms affixes', cf. Zribi-Hertz, 1997; Culbertson, 2010) générés par des têtes fonctionnelles, ce qui ferait du créole haïtien une langue à sujet nul. En revanche, d’après Déprez (1994), Baptista (1995) et Cadely (1995, 1997) ce sont des pronoms avec un statut d'argument positionné en surface dans specIP (Spécifieur du Syntagme Flexionnel IP dominant le Syntagme Verbal) mais réduits au niveau phonologique seulement. En ce qui concerne les clitiques objets du créole haïtien, ils apparaissent systématiquement dans la même position syntaxique que l'argument correspondant exprimé par un SN et ont aussi été analysés comme des clitiques phonologiques, mais pas syntaxiques (Lefebvre, 1998 ; Guijarro-Fuente \& Ortiz López, 2008). Il est à noter, que toutes ces analyses du créole haïtien, sauf celles de Guijarro-Fuente \& Ortiz López (2008) et de Valdman (2015) se fondent sur les jugements de grammaticalité de quelques locuteurs natifs (souvent l'auteur même de l'étude, e.g. DeGraff, 1993). Par rapport aux démarches effectuées par les auteurs d'études récentes du français, celles-ci n’ont pas été mises à l'épreuve de l'analyse de corpus. La présente étude a donc pour objectif de comparer les statuts syntaxiques et phonologiques des pronoms Personnels du créole haïtien et du français en se fondant sur des analyses de corpus d'échantillons de parole spontanée.

$\underline{\text { Tableau } 2 \text { : Paradigme des pronoms Personnels et possessifs et déterminants possessifs en créole haïtien }}{ }^{4}$

\begin{tabular}{|c|c|c|c|c|c|c|c|}
\hline \multirow[t]{2}{*}{ Nombre } & \multirow[t]{2}{*}{ Personne } & Sujet & $\begin{array}{l}\text { Objet } \\
\text { Direct }\end{array}$ & $\begin{array}{l}\text { Objet } \\
\text { Indirect }\end{array}$ & Réfléchis & $\begin{array}{l}\text { Pronom } \\
\text { Possessifs }\end{array}$ & $\begin{array}{l}\text { Déterminants } \\
\text { Possessifs }\end{array}$ \\
\hline & & SPn & DOPn & IOPn & REFPn & POSPn & POSPn \\
\hline \multirow[t]{3}{*}{ SG } & $1^{\text {ère }}$ & mwen/m* & mwen/m & mwen/m & mwen menm & pa mwen/m & mwen/m \\
\hline & $2^{\text {ème }}$ & $\mathrm{ou} / \mathrm{W}$ & $\mathrm{ou} / \mathrm{W}$ & $\mathrm{ou} / \mathrm{w}$ & ou menm & pa ou/w & $\mathrm{ou} / \mathrm{W}$ \\
\hline & $3^{\text {ème }}$ & $\mathrm{li} / \mathrm{l}$ & $\mathrm{li} / \mathrm{l}$ & $\mathrm{li} / \mathrm{l}$ & li menm & pa li/l & $\mathrm{li} / \mathrm{l}$ \\
\hline \multirow[t]{3}{*}{ PL } & $1^{\text {ère }}$ & nou/n & nou/n & nou/n & nou menm & pa nou/n & nou/n \\
\hline & $2^{\text {ème }}$ & nou/n & nou/n & nou/n & nou menm & pa nou/n & nou/n \\
\hline & $3^{\text {ème }}$ & yo/y & yo/y & yo/y & yo menm & pa yo/y & yo/y \\
\hline
\end{tabular}

*Pour chaque pronom, la version phonologique pleinement réalisée est indiquée à gauche et la forme réduite à droite.

\section{Approche Méthodologique}

\subsection{Introduction}

Les premières analyses qui ont rendu compte des distinctions des pronoms forts et faibles ou clitiques du français s’appuyaient sur les jugements de grammaticalité des auteurs (e.g. Kayne, 1977 ; Ruwet, 1976) qui avaient tendance à refléter la langue écrite standard. Depuis les années 80 et les travaux pionniers de 
Blanche-Benveniste et ses collègues dans ce domaine (e.g. Blanche-Benveniste \& Jeanjean, 1987), de nombreux corpus du francais oral représentant plusieurs registres y compris la parole spontanée ont été mis à la disposition des chercheurs y compris les corpus CHILDES incluant la parole adulte addressée à l'enfant (MacWhinney, 2000) pour les études d'acquisition du langage (Bassano, 1988, Pierce, 1992 ; Culbertson \& Legendre, 2008 ; Culbertson, 2010), d'autres permettant la comparaison de différentes variétés du français (e.g. Auger, 1995, 2003 ; Detey et al., 2010). Les corpus de parole courante spontanéee ont révélé que ses caractéristiques sont typiquement différentes du francais soutenu, non seulement au niveau lexical mais aussi grammatical (e.g. Totereau, Thévenin \& Fayol, 1997). L’accès aux enregistrements a aussi permis des analyses acoustiques qui ont parfois remis en question les premières analyses de la distribution des pronoms forts et faibles coréférents. Par exemple, la combinaison d'un SN et d'un clitique renvoyant à un même référent a été interprétée comme une dislocation par de nombreux auteurs (e.g. Kayne, 1977; Rizzi, 1986) qui pensaient que de telles constructions contenaient systématiquement une pause prosodique. Les analyses acoustiques d'échantillons de parole spontanée ont permis de conclure que ce n'était pas le cas et que la coocurrence du SN ou pronom fort ne met pas en jeu une pause (indiquée par la virgule dans l'exemple 9a) et constitue le plus souvent un exemple de «redoublement du SN par un clitique » comme 9b (cooccurrence d'un clitique et d'un SN ou pronom fort qui font référence à la même entité). D’après les données expérimentales du registre courant spontané (Culbertson \& Legendre, 2008 ; Culbertson, 2010) la dislocation d'un SN objet porte la signature prosodique distinctive (Doetjes et al., 2002) d'une dislocation en français alors que la combinaison d'un SN suject et d'un clitique sujet ont une prosodie identique à celle du seul SN sujet en français standard en 9c.

(9) a Pierre , $_{\mathrm{i}} \mathrm{il}_{\mathrm{i}}$ entend

(9) b Pierre ${ }_{i} i_{i}$ entend

(9) c Pierre entend

\subsection{Sélection et création de corpus}

Ce projet s'inscrit dans le cadre d'une étude comparative des pronoms sujets et marques d'accord chez les enfants apprenant le français ou le créole haïtien comme première langue. Les études comparatives de l'acquisition des langues ont révélé des profils développementaux différents chez les enfants qui acquièrent des langues à sujet nul et à sujet obligatoire (e.g. Valian, 1991) et pour l'acquisition des pronoms forts et faibles dans une même langue (e.g. Pierce, 1992 ; Jacubowicz, 1997 ; Legendre et al., 2010). Depuis les années 80, l’accès aux corpus de parole spontanée (MacWhinney, 2000) a permis aux psycholinguistes s'intéressant à l'acquisition de déterminer plus précisement les caractéristiques des langues auxquelles les enfants sont exposés lorsqu'ils l'acquièrent comme première langue. Le projet que nous évoquons ci-dessous dispose d'un grand nombre de corpus pour l'acquisition du français, y compris sur la base de données CHILDES (MacWhinney, 2000). Ceci n’est pas le cas pour le créole haïtien. A notre connaissance, seul un corpus de parole spontanée a été établi et il ne répond pas aux besoins de notre étude. Il s’agit de celui de Valdman (2015), recueilli au Cap Haïtien dont la variété dialectale diffère de celle de Port-au-Prince (dite variété standard et qui fait l'objet de notre étude) par un certain nombre de traits syntaxiques, y compris la réalisation du pronom de la troisième Personne du singulier. Afin de pallier à cette quasi-inexistence de corpus, nous avons établi un premier corpus de créole haïtien standard (Barrière, 2015) qui est en train de nous permettre de développer des outils d'analyse. Il a été enregistré au sein de la communauté haïtienne de New York qui compte un nombre important de locuteurs : le créole haïtien se range en quatrième position après l'espagnol, le chinois et le russe en nombre de Personnes utilisant une langue autre que l'anglais à la maison (Barrière \& Monéreau-Merry, 2015). Pour les locuteurs qui ont participé au développement de ce corpus, il s’agit de la langue vernaculaire dominante dans de nombreux contextes, y compris leurs foyers et de nombreux espaces culturels comme les émissions de radio. Le corpus comprend des échantillons de parole spontanée de cinq locuteurs natifs du créole haïtien standard engagés dans un débat animé lors d'une émission radiophonique (Corpus HC radio) et de parents (un père et une mère) dans un contexte d'interaction avec un jeune enfant (Corpus HC 
Parents). Il est à noter que le corpus radiophonique ne comprend pas l'enregistrement de l'animateur au début de l'émission qui est d'un niveau de parole plus soutenu que le débat qui suit. La transcription commence au moment où des auditeurs appellent pour intervenir spontanément sur les thèmes soulevés par l'émission qui a trait à l'évolution de l'espèce humaine et la religion- sujets qui provoquent de nombreuses réactions et désaccords chez les locuteurs. Si l’on applique la classification de Labov (1966) aux deux échantillons de parole enregistrés dans les contextes domestiques et radiophoniques, les deux correspondent à la parole courante spontanée ('casual speech' selon Labov) et pas au registre soutenu ('careful speech' selon Labov), de par les caractériques grammaticales et morphophonologiques des énoncés ainsi que de leur débit rapide et l'organisation de la prise de parole. Ces deux échantillons représentent donc le même registre de parole courante spontanée que les corpus oraux analysés par Culbertson et Legendre (2008) pour le français.

Le premier corpus (contexte radiophonique) contient un total de 574 énoncés et le second (contexte domestique) 330 énoncés. Les deux enregistrements ont été transcrits dans le format CHAT (MacWhinney, 2000) par une locutrice native du créole haïtien et vérifiés par deux autres locuteurs natifs. Ce corpus contient assez de structures syntaxiques variées, pour nous permettre de développer un outil MOR qui utilisé avec le programme CLAN (MacWhinney, 2000) permet une analyse automatique des phrases. Etant donné le syncrétisme des pronoms et déterminants possessifs (voir tableau 2), les pronoms ont été transcrits avec un chiffre qui les suit et qui permet de différencier rapidement les pronoms sujets de pronoms objets directs et indirects et des déterminants possessifs afin d'extraire les exemples du corpus.

\section{Les pronoms sujets}

\subsection{Les pronoms sujets du français}

Au-delà des propriétés mentionnées ci-dessus les clitiques sujet du français ont un certain nombre d'autres propriétés phonologiques et syntaxiques qui sont répertoriées ici à la lumière des travaux récents de Culbertson et Legendre (2008) et Culbertson (2010). Dans le registre courant spontané le clitique sujet est soumis à des processus phonologiques qui sont spécifiques aux affixes et aux clitiques, et dans certains cas à des combinaisons d'un clitique sujet + verbe spécifiques (Culbertson, 2010; FonsecaGreber et Waugh, 2002; Miller, 1991; Morin, 1979). C’est le cas, par exemple, de la fusion entre je et suis ([ $\left.\int \mathrm{syi}\right]$; à l'infinitif: être) par contraste avec l'absence de fusion avec le verbe homophone dans je suis ([3əsyi] ; à l'infinitif: suivre). Un autre processus phonologique à l'oeuvre en présence d'un clitique sujet concerne sa réduction (obligatoire) par la perte de la voyelle finale ou médiale devant un verbe commençant par une voyelle (j'arrive $=>$ [3ariv]) et la perte (facultative) d'une consonne finale devant un verbe commençant par une consonne (il part => [ipar], [ilpar]. Alors que l'élision de la voyelle se fait en présence d'un clitique sujet ou d'un article défini, l'élision de la la consonne est restreinte au seul clitique sujet. Selon Plénat (1986) et Miller (1991) l'enchaînement associé à l'élision ou la liaison est synonyme d'attachement lexical et elle concerne les clitiques (y compris les articles) et les affixes en français. La liaison de la consonne nasale [n] est obligatoire pour le clitique sujet on et les affixes en- et un- (voir $10 \mathrm{a}, \mathrm{b})$ et interdite dans d'autre contextes, tels que 10c..

(10) a. on ira $\Rightarrow[$ õnira $] / *[$ õira $]$

b. enivrer $\Rightarrow$ [ãnivre] $/ *[a ̃ i v r e]$

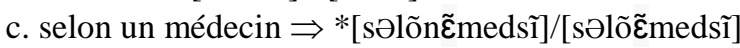

Le fait que le marqueur de portée négative ne intervienne entre le clitique sujet et le verbe, mais pas entre le clitique objet et le verbe (je ne l'ai pas vue), est traditionellement interprété comme indice de la position structurale plus élevée des clitiques sujets dans une représentation de type arbre syntaxique, comparée à celle plus basse des clitiques objets et donc étaye en principe l'hypothèse que le clitique sujet n'a pas le statut d'affixe en français (Rizzi 1986). Cette conclusion s'appuie sur le fait que ne n'a pas luimême le statut d'affixe car il peut dépendre d'un adverbe (Elle dit ne souvent pas être satisfaite). A 
l'examen de corpus de français parlé (Ashby, 1981; Coveney, 2002) ne se révèle cependant être en perte d'usage à des taux variables. Les différences de proportion d'absence de ne sont probablement dûes aux différences de registres utilisés dans les différent corpus et même mixtes au sein même d'un corpus (Culbertson \& Legendre, 2008). Dans la parole adressée à l'enfant (corpus de Lyon; Demuth \& Tremblay, 2008) ne est systématiquement omis en présence de sujets négatifs comme en 11a et pas marque la portée négative comme en 11b.

\section{(11) a. Personne m'en a parlé. (Nat25) \\ b. Tu essaies de pas colorier sur le jaune. (Tim20)}

Bien que le taux de rétention de ne dans l'ensemble du corpus de Lyon est de 7\%, c'est sa distribution qui est révélatrice du statut du clitique sujet. Le taux de rétention de ne dépend du type de sujet présent (Tableau 3; Culbertson, 2010). La rétention est la plus élevée lorsqu’il s’agit d’un SN sujet (y compris les sujets négatifs), suivi par les sujets ‘nuls’ (impératif, y’a existentiel, faut). La rétention est la moins élevée en présence d'un clitique sujet. Donc ne est de préférence absent précisément dans les contextes où il interviendrait entre le clitique sujet et le verbe. La distribution de ne se révèle donc étayer l'hypothèse inverse selon laquelle le clitique sujet a le statut d’affixe dans le registre oral spontané.

Tableau 3 : Proportions de rétention de ne en parole spontanée (Corpus de Lyon, Culbertson, 2010)

\begin{tabular}{llll} 
& nе... & & Total \\
\hline \hline SN sujet & $83,3 \%$ & 20 & 24 \\
Absence de SN/clitique sujet & $14,1 \%$ & 104 & 737 \\
Clitique sujet & $6,3 \%$ & 323 & 5092 \\
SN + clitique sujet & $6,7 \%$ & 9 & 137 \\
Total & $7,6 \%$ & 456 & 5990 \\
\hline
\end{tabular}

Dans le registre écrit standard les clitiques sujets subissent l'inversion dans les questions des types 12a et $12 \mathrm{~b}$.

$$
\begin{aligned}
& \text { a. Veut-il du café ? } \\
& \text { b. Que veut-il? }
\end{aligned}
$$

Mais la fréquence de l'inversion dans les corpus de parole spontanée entre adultes est extrêmement peu élevée ( $0 \%$ dans les questions oui-non vs. 2\% dans les questions wh; De Cat, 2007). Les taux sont encore plus bas dans le corpus de Lyon (Culbertson \& Legendre, 2008; Culbertson, 2010): 0\% dans les questions oui-non (0/2811) versus 0,9\% dans les questions wh (13/1388). La distribution de l'inversion est donc identique à celle de ne ; les deux montrent que la grammaire du français oral spontané est sensible au statut affixal, plus précisément préfixal, du clitique sujet.

Lorsque deux verbes sont coordonnés, la répétition du clitique sujet est traditionellement caractérisée comme non-obligatoire en français. Mais dans le corpus de Lyon, la répétition du clitique sujet est quasi-obligatoire (98,9\%, 180/183; Culbertson et Legendre, 2008), ce qui est prédit par l'analyse affixale du clitique sujet. A noter une différence entre la parole spontanée en 13a et la parole lue à partir de livres pour enfants $(38 \%, 14 / 37)$ en 13b. Cette dernière reflète le registre écrit standard de la langue.

(13) a Il va ouvrir la porte et il va rentrer. (Nat1)

b Elle déposa son panier dans l'herbe et se mit à cueillir des fleurs. (Nat31)

Quant au redoublement des SN non-quantifié en fonction sujet par un clitique comme en 14a, ils sont presque toujours redoublés dans les énoncés déclaratifs du corpus de Lyon (85\%, 154/182).

(14) a. Jean il parle. 


\section{b. Personne $(* \mathbf{i l})$ ne parle.}

Le redoublement n’étant en général pas permis en français avec les sujets indéfinis ou quantifiés (14b) ce que Culbertson et Legendre (2008) attribuent à l'absence d'appariement de traits- la fréquence du redoublement du sujet [+défini] est même plus élevée: 89\% (154/174) (voir aussi la discussion des contraintes sémantiques dans les constructions SPn+ SN+SCL de Blasco-Dulbeco \& Caddeo, 2002). Cette proportion n'inclut pas les pronoms forts qui eux ont un taux de redoublement de presque $100 \%$ (588/589). En comparaison, la fréquence du redoublement chez 23 locuteurs adultes de Paris et Lyon du corpus PFC (Phonologie du français contemporain, Durand et al. 2002, 2005) est de 60\% avec les SN en fonction sujet mais dépend aussi de l'âge des locuteurs.

L’analyse syntaxique de la construction illustrée en 14a est au centre du débat concernant le statut du clitique sujet. S'il s'agit de redoublement du SN alors le clitique lui-même ne peut avoir de statut autre que celui d'affixe flexionnel. Par contre, si 14a est un exemple de dislocation (à gauche) du SN sujet, alors le clitique peut en principe occuper la position sujet (Spécifieur de la tête flexionelle, specIP). Le contraste en 14 est fréquemment cité comme preuve que la construction en 14a est un cas de dislocation et non un cas de redoublement. Puisque les SN quantifiés ne sont jamais des topiques, ils ne peuvent pas être disloqués; ils doivent occuper la position sujet. Les SN non-quantifiés tels que Jean en 14a ne sont pas soumis à la même restriction. Lorsque le sujet est un topique, il est disloqué et specIP est occupé par un pronom résomptif. L'apparition du clitique correspond donc à la possibilité de disloquer le sujet (Rizzi, 1986 ; De Cat, 2007). En l'absence de dislocation, le pronom clitique et le SN sont en compétition pour la même positionsujet. Il en résulte deux possibilités (Jean parle, Il parle).

Il est en général admis que dans les énoncés à focus ce dernier est en position sujet en français (e.g. Samek-Lodovici, 2005) mais identifiés comme focus large ou focus étroit par une prosodie spécifique (Marandin, 2004). Il est à noter que le redoublement clitique est obligatoire dans le registre oral en présence d'un pronom fort de la première ou deuxième Personne (moi, toi), comme le montre 15a, même lorsqu'il est interprété comme focus étroit, comme en 15b.

(15) a. Moi je parle beaucoup/* Moi parle beaucoup.

b. Qui a appelé Jean ? Moi je l’ai appelé/* Moi l’ai appelé

Fonseca-Greber \& Waugh (2002) n’identifient aucun exemple de pronom fort sans redoublement clitique dans leur corpus de français oral contemporain, et le corpus de Lyon ne présente qu'un seul exemple de la troisième Personne (lui) sans redoublement clitique parmi 589 exemples de pronoms forts (Culbertson, 2010). Le redoublement clitique obligatoire en présence d'un pronom fort et la possibilité de redoublement clitique dans les énoncés à focus montrent bien que les clitiques sujet peuvent en fait coexister avec un SN dans une projection flexionelle de type IP, et donc qu'ils ne sont pas en compétition avec le sujet nominal en ce qui concerne la position specIP et le rôle d'argument qui y correspond.

\subsection{Les pronoms sujets du créole haïtien}

L'un des comportements clés qui distingue les pronoms forts des clitiques syntaxiques en français est le fait que ces derniers ne peuvent pas être conjoints (voir exemples 4a,b,c ci-dessus). En revanche, le comportement des pronoms sujets en créole haïtien s'apparente à celui des pronoms forts puisqu'ils peuvent être conjoints avec un SN lexical, ce qui rend compte de la grammaticalité de 16.

nou avèk Jean-Beliard-Lucien bò tab la (Corpus HC Radio) SPn-PL2 CONJ- avèk N- Jean-Beliard-Lucien PREP- bò N-tab Def-Det-SG nous et Jean-Beliard-Lucien (sommes) à table

Tandis que dans le registre spontané du français les clitiques sujets ne peuvent être séparés de leur hôte que par d'autres clitiques et affixes, autrement dits des éléments qui relèvent de catégories flexionnelles, 
les pronoms sujets haïtiens peuvent être séparés de leur hôte par différents types d'éléments lexicaux. Par exemple ils peuvent être séparés par des adverbes, comme les exemples 17a et17b le montrent.

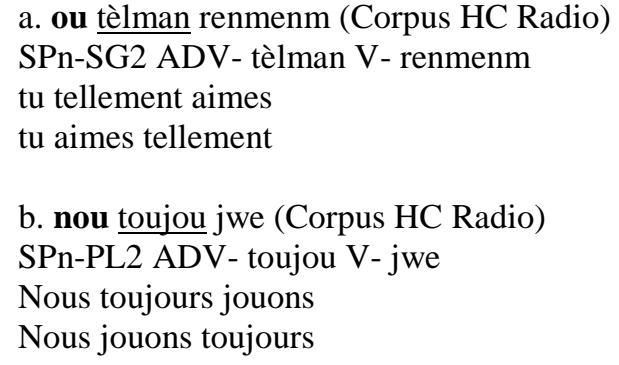

En ce qui concerne les marqueurs de négation du créole haïtien comme pa 'ne-pas' et ni..ni 'ne...ni' (soulignés dans les exemples ci-dessous), ils suivent toujours le pronom sujet et apparaissent donc entre celui-ci et le marqueur de Voix, Temps et Aspect, comme dans 19a, ou entre le pronom sujet et le verbe, comme dans 18a et 20a, ce dernier contenant trois exemples de négation. Ce principe rend compte de la grammaticalité de 18a,19a et 20a et de l'agramaticalité de 18b, 19b et 20b : les marqueurs de portée négatives ne peuvent pas apparaître après le verbe. Cette distribution des marqueurs de négation est très différente des observations effectuées sur les corpus de français spontané décrites dans la section 3.1. L'exemple 20a nous permet aussi d'explorer un autre phénomène, celui de la conjonction de plusieurs verbes, autre domaine dans lequel les faits que nous observons en créole haïtien diffèrent de ceux rapportés pour le français spontané. Les deux verbes kraze 'écraser' et diminye 'diminuer' ont tous les deux pour sujet le pronom $m$ (wen) qui apparait devant le premier verbe et n’est pas répété devant le deuxième. Cet énoncé a été produit dans le contexte d'un débat animé. Il s’agit donc d'un phénomène différent de ce qui a été rapporté pour le français dans lequel ce type de construction relève de la langue écrite standard (voir la discussion des exemples 13a et 13b dans la section 3.1).

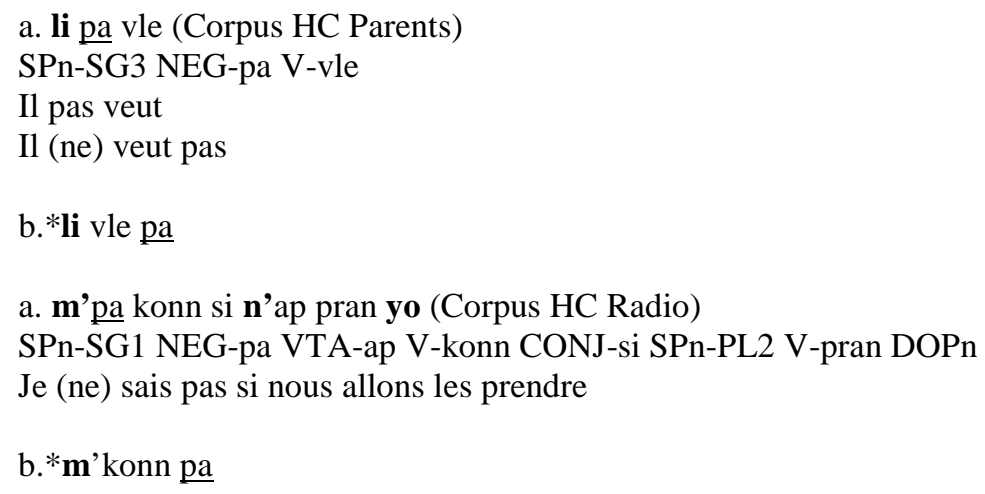

(20) a. sa m'pa pran'l pou on fason pou'm ni kraze ni diminye (Corpus HC Radio) DEMPn SPn-SG1 NEG-pa V-pran DOPn-SG3 CONJ-pou QUANT-on N-fason SPnSG1 NEG-ni V-kraze NEG- diminye

Cela je (ne) le prends pas pour une manière (pour moi/de ma part) d'écraser ou de diminuer

b.*pou'm kraze ㅆi diminye $\underline{\text { ni }}$

Comme il est expliqué dans la section 3.1, tandis que les analyses du français ont tendance à analyser les combinaisons de clitique sujet et pronom fort ou SN comme des exemples de dislocation, les études de corpus et les analyses de Culbertson (2010) montrent sur la base d'études de corpus et d'études 
expérimentales que ces cooccurrences sont extrêmement fréquentes dans le registre spontané du français et qu'elles constituent le plus souvent des cas de redoublement de clitique, pas de dislocation. Dans nos deux corpus de créole haïtien, nous n'avons trouvé qu’un seul énoncé qui contienne un sujet clitique et un SN coréférents qui est reproduit en 21.

(21) a. E nati $a_{i}, \mathbf{l i}_{\mathbf{i}}$ genyen tout sa'l bezwen yo pou'l funksyone (Corpus HC Radio) CONJ-e N-nati DefDet-Sg, SPn-SG3 a QUANT-tout DEMPn-sa- SPn-SG3 V-bezwen DOPn-PL3 CONJ-pou- SPn-SG3 V- funksyone

Et la nature, elle a tout ce dont elle a besoin pour fonctionner

b. * E nati a l(i) genyen tout sa

c. Sa'l santi ?

WH-Q SPn-SG3 V-santi?

Que ressent- il?/ Qu'est-ce qu'il ressent?

L'exemple 21a contient une virgule marquant une pause, ce qui fait de cette construction une dislocation. Les possibilités de réduction phonologique des pronoms sujets en créole haïtien permettent de déterminer si le redoublement de clitique est possible dans cette construction. Comme il est expliqué ci-dessous (voir discussion des exemples 23 et 24), le créole haïtien permet la procliticisation, c'est-à-dire l'agglutination du clitique au mot qui le suit (comme le permet le français). A la différence du français, il permet aussi l'encliticisation, c'est-à-dire l'agglutination au mot qui le précède : ceci explique la grammaticalité de l'exemple 21c. [Afin de tester si le redoublement de clitique est possible lorsqu'il met en jeu un pronom sujet, il suffit d'évaluer la grammaticalité de l'agglutination du pronom à l'élément linguistique qui le précède mais en l'absence d'une pause]. Comme le montre l'exemple 21b, l'élimination de la pause et l'encliticisation engendrent une construction que deux locuteurs natifs considèrent agrammaticale. Cela nous conduit à conclure que, contrairement à ce qu'affirme Saint Martin (2003) dont les propos à ce sujet sont ambigus car il évoque la dislocation mais fournit des exemples de redoublement de clitique, le comportment des pronoms sujets en créole haïtien diffère du français. Premièrement, les co-occurrences de $\mathrm{SN}$ et pronoms sujets sont rares et la répétition du pronom sujet inexistante dans notre corpus (sauf dans deux contextes d'hésitation), alors qu'en français cette cooccurrence est extrêmement fréquente (voir section 3.1, discussion des exemples 14 et 15). Ensuite, tandis que la plupart de ces cooccurrences en français oral spontané ne mettent en jeu aucune pause et constituent des cas de redoublement de clitique, ce type de construction est agrammatical en créole haïtien. Les rares cas de cooccurrence de SN et pronom sujet sont vraisemblablement des cas de dislocation.

Dans toutes les phrases de notre corpus qui contiennent un prédicat (c'est-à-dire un total de 1074 syntagmes verbaux), le sujet est exprimé, sauf dans les constructions impératives et celles qui mettent en jeu un verbe existentiel qui permet l’omission du sujet non-référentiel (Déprez, 1994; Baptista, 1995), comme dans l'exemple 22. Le créole haïtien ne se comporte donc pas comme une langue à sujet nul typique.

$$
\begin{aligned}
& \text { Li prouve ø gen zosman dinozò yo sou tè a (Corpus HC Radio) } \\
& \text { SPn-SG3 V-prouve V-a N-zosman N- dinozò Def-Det-PL PREP-sou tè Def-Det-SG } \\
& \text { Il prouve } \emptyset \text { y-a os dinosaures les sous terre la } \\
& \text { Cela prouve (qu'il) y-a des os des dinosaures sous la terre }
\end{aligned}
$$

Comme la section 3.1 ci-dessus l'explique, les réductions des clitiques du français sont soumises à de nombreuses contraintes phonologiques, grammaticales et lexicales. En revanche, lorsque les pronoms sujets du créole haïtien apparaissent réduits au niveau phonologique, leur réduction et leur restructuration syllabique met en jeu des éléments linguistiques variés, y compris des éléments autres que le verbe et qui peuvent précéder ou suivre le pronom. Autrement dit, la réduction phonologique peut metttre en jeu des éléments avec lesquels le pronom n’entretient pas de relation de dépendance syntaxique et le résultat est 
une procliticisation ou encliticisation au niveau phonologique. Il n'existe pas de restriction de nature syntaxique en ce qui concerne l'hôte du pronom sujet tandis que des contraintes phonologiques supplémentaires s’appliquent (Cadely, 1995, 1997). Les exemples 23 et 24 illustrent le phénomème de réduction et de restructuration avec les élements qui précèdent ou suivent. Ainsi, dans l'exemple 23, le pronom sujet de la première Personne du singulier [mwĩ] est réduit et s'agglutine au marqueur de Temps, Aspect et Voix qui le suit : il s'agit donc d'un proclitique. Dans la même phrase, le pronom objet de la troisième Personne du singulier [li] est aussi réduit mais il s’agglutine à l'élément qui le précède : cet exemple est donc un exemple d'enclitique. Un des points communs à ces deux exemples de réduction est que les deux pronoms s'agglutinent aux éléments linguistiques avec lesquel ils entretiennent une relation de dépendence, mais les principes de l'agglutination n’y sont en général pas contraints. Ainsi l'exemple 24 contient deux enclitiques qui constituent des cas de réduction de la troisième Personne du singulier: le deuxième et le quatrième pronom de la phrase. Au niveau phonologique les deux s'agglutinent à des élements qui les précèdent avec lesquels ils n’entretiennent pas de relation de dépendence syntaxique. Ainsi dans le cas de $s a$ 'l, le pronom [li] est réduit et s'agglutine à l’objet (exprimé par le pronom démonstratif) du verbe de la phrase principale alors qu'au niveau syntaxique, il s'agit du sujet du verbe de la phrase enchâssée bezwen 'avoir besoin', qui le suit. De même le dernier pronom [li] s'agglutine à la conjonction pour 'pour que' (pou'l) tandis qu'au niveau syntaxique il s'agit du sujet du verbe funksyone 'fonctionner', qui le suit.

(23) Kounyea m'pral lague'l nan on pwoblèm timoun lan na on lekòl [e] [e] katolik (Corpus HC Radio)

ADV- Kounyea SPn-SG1-VTA-pral _V-lague-DOPn-SG3 PREP-nan on-QUANT Npwoblèm N-timoun Def-Det-SG PREP-na on-QUANT [euh] [euh] N- lekòl ADJkatolik

Maintenant je vais créer un problème pour cet enfant qui est dans une école catholique.

(24) E nati a, li genyen tout sa'l bezwen yo pou'l funksyone (Corpus HC Radio)

CONJ-e N-nati DefDet-Sg, SPn-SG3 a QUANT-tout DEMPn-sa- SPn-SG3 V-bezwen

DOPn-PL3 CONJ-pou- SPn-SG3 V- funksyone

Et la nature, elle a tout ce dont elle a besoin pour fonctionner

Afin de déterminer si ces phenomènes de réduction, tous facultatifs en créole haïtien, sont rares ou fréquents nous avons effectué une analyse quantitative dont les résultats sont présentés dans le tableau 4 ci-dessus. Elle révèle que quel que soit le contexte des enregistrements (conversation entre adultes ou parents s'adressant à un enfant) les contractions existent mais leur taux de fréquence varie: elles constituent entre $20 \%$ et $100 \%$ de la réalisation des pronoms. Certains, comme ceux de la première Personne du singulier tendent à être plus fréquemment réduits que les autres (Tableau 4), dans les deux corpus. Ainsi le pronom sujet de la première Personne du singulier apparait plus fréquemment sous sa forme réduite tandis que la forme pleinement réalisée du nou qui indique les première et deuxième Personnes du pluriel est plus fréquente que la forme réduite.

Notre comparaison des pronoms sujets du français et du créole haïtien fondée sur les analyses de corpus de parole spontanée mettent en lumière des contrastes importants tant au niveau syntaxique que phonologique, sur lesquels nous revenons dans la conclusion (section 5) ci-dessous, après avoir traité des pronoms objets. 
Tableau 4: La distribution des différentes réalisations phonologiques des pronoms sujets

\begin{tabular}{|c|c|c|c|}
\hline & CORPUS 1 & CORPUS 2 PARE & \\
\hline & $\begin{array}{l}\text { HC PROGRAMME DE } \\
\text { RADIO }\end{array}$ & MÈRE-ENFANT & PÈRE-ENFANT \\
\hline Pronoms Sujets & $362(100 \%)$ & $48(100 \%)$ & $14(100 \%)$ \\
\hline $1^{\text {ère }}$ Personne du Singulier MWEN & $74(21 \%)$ & $2(4 \%)$ & $6(43 \%)$ \\
\hline Forme pleine MWEN & $15(20 \%)$ & $0(0 \%)$ & $0(0 \%)$ \\
\hline Forme réduite $\mathrm{M}$ & $59(80 \%)$ & $2(100 \%)$ & $6(100 \%)$ \\
\hline $2^{\mathrm{ND}}$ Personne du singulier $\mathrm{OU}$ & $103(29 \%)$ & $41(85 \%)$ & $4(29 \%)$ \\
\hline Forme pleine OU & $61(59 \%)$ & $26(63 \%)$ & $4(100 \%)$ \\
\hline Forme réduite $\mathrm{W}$ & $42(41 \%)$ & $15(37 \%)$ & $0(0 \%)$ \\
\hline $3^{\text {ème }}$ Personne du Singulier LI & $48(13 \%)$ & $1(2 \%)$ & $4(29 \%)$ \\
\hline Forme pleine LI & $28(58 \%)$ & $0(0 \%)$ & $3(75 \%)$ \\
\hline Forme réduite $\mathrm{L}$ & $20(42 \%)$ & $1(100 \%)$ & $1(25 \%)$ \\
\hline $1^{\text {ère }} / 2^{\text {ème }}$ Personne du Pluriel NOU & $113(31 \%)$ & $3(6 \%)$ & $\mathbf{0}(\mathbf{0} \%)$ \\
\hline Forme Pleine NOU & $76(67 \%)$ & $3(100 \%)$ & $0(0 \%)$ \\
\hline Forme réduite $\mathrm{N}$ & $37(33 \%)$ & $0(0 \%)$ & $0(0 \%)$ \\
\hline $3^{\text {eme }}$ Personne du Pluriel YO & $24(7 \%)$ & $1(2 \%)$ & $0(0 \%)$ \\
\hline Forme pleine YO & $19(79 \%)$ & $1(100 \%)$ & $0(0 \%)$ \\
\hline Forme réduite Y & $5(21 \%)$ & $0(0 \%)$ & $0(0 \%)$ \\
\hline
\end{tabular}

\subsection{Les pronoms objets du créole haïtien}

Les pronoms objets du créole haïtien diffèrent des clitiques objets du français quant à leurs formes et leur distribution syntaxique. Comme le tableau 2 du paradigme de ces pronoms le montre, les pronoms objets et sujets sont caractérisés par leur syncrétisme à toutes les Personnes, comme l'illustre l'exemple 25. La distinction entre les deux cas est exprimée par la position des pronoms. (25) Yo wè yo (HC Radio Program)

(25) Yo wè yo (HC Radio Program)

SPn-PL3 V- wè DOPn-PL3

Ils les voient

De plus, l'objet indirect apparaît avant l'objet direct, ce qui rend compte de la grammaticalité de 26 tandis que l'ordre inverse engendre une phrase agrammaticale.

(26) a. ou te di m'li (HC Radio Program)

SPn-SG2 VTA-te V- di IOPn-SG1-DOPn-SG3

Tu me l'as dit

b.*Ou te di li m(wen)

Alternativement, une préposition peut être utilisée afin d'indiquer des distinctions de cas, comme dans l'exemple 27 ci-dessous.

(27) Kom Jean-Beliard (ap) (ap) ap defile'l pou nou la a (HC Radio Program)

CONJ-kom V-Jean-Beliard VTA-ap V-defile-DOPn-SG3 PREP-pou IOPn-PL1 ADV-la

Comme Jean-beliard va l'énumerer pour nous ici

(28) a Nou kontrole'l (HC Radio Program)

SPn-PL1 V-kontrole-DOPn-SG3

Nous le contrôlons 
b *Nou l(i) kontrole

(29) Mwen wè'l ak Jean-Beliard (HC Radio Program) SPn-SG1 V-wè-DOPn-SG3 CONJ-ak Jean-Beliard Je (le) vois lui avec Jean-Beliard

En ce qui concerne leur distribution syntaxique, comme les exemples 25, 26, 27, 28 et 29 ci-dessus l'illustrent, les pronoms objets en créole haïtien apparaissent toujours après le verbe, c'est-à-dire dans la même position que l'argument auquel ils se référent lorsqu'il est exprimé par un SN lexical, ce qui rend compte de l'agrammaticalité de 28b. Comme les pronoms sujets, ils peuvent être coordonnés, comme l'illustre la grammaticalité de l'exemple 29 Ces deux comportements montrent qu'ils ont plus de traits en commun avec les pronoms objets forts qu'avec les clitiques objets du français. Comme les exemples 26a, 27, 28a et 29 le montrent, ils peuvent être réduits au niveau phonologique.

En ce qui concerne la cooccurrence de pronoms objets et de SN coréférents, aucun exemple n’a été identifié dans nos deux corpus. Afin de vérifier si l'absence de ces constructions dans notre corpus reflète des faits qui relèvent de la grammaire du créole haïtien ou de facteurs dus à l'échantillonage de nos enregistrements, tels que le nombre relativement limité d'énoncés ou les contextes d'interaction, nous avons eu recours à des jugements de grammaticalité par deux locuteurs natifs. Pour aucun d'entre eux, le redoublement du pronom sujet comme dans 30a n'est jugé acceptable. L'exemple de dislocation illustrée par 30b, c'est-à-dire l'occurrence du SN après le pronom sujet suivi d'une pause, est considérée plus acceptable que le redoublement en 30a mais pas complètement. En revanche, 30c qui illustre un cas de topicalisation est grammaticale.

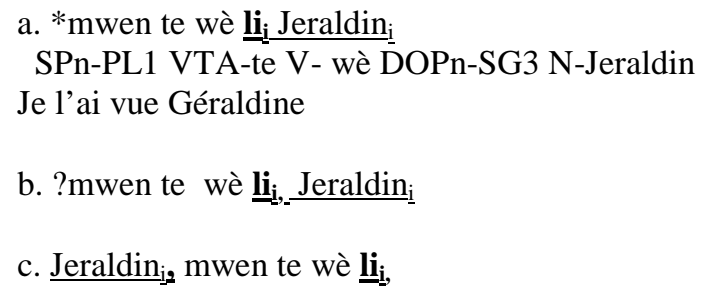

Les faits ci-dessus montrent que la distribution syntaxique des pronoms objets du créole haïtien diffère de la distribution des clitiques objets du français dans plusieurs domaines, y compris leur position vis-à-vis du verbe et l'agrammaticalité du redoublement du pronom objet et du SN.

Comme le montrent les exemples 27, 28 et 29 ci-dessus les pronoms objets du créole haïtien, tout comme les pronoms sujets, peuvent être réduits au niveau phonologique. Ainsi l'exemple 26a présente un cas de proclitique : le pronom objet direct est agglutiné au pronom objet indirect. Et les exemples 27, 28 et 29 contiennent des exemples d'enclitique : les pronoms objets directs sont agglutinés aux verbes qui les précèdent.

\section{Conclusions}

Notre comparaison des pronoms sujets du français et du créole haïtien fondée sur les analyses de corpus de parole spontanée mettent en lumière des contrastes importants. Contrairement aux clitiques sujets du français qui ont le status d'affixe dans le registre spontané de la langue, les pronoms sujets du créole haïtien oral se comportent comme des SN arguments quant à leur possibilité de co-ordination, dislocation, conjonction, et séparation d’avec le verbe dont ils dépendent. Dans les deux langues, la réduction phonologique entraîne l'agglutination à un hôte. En français, cette réduction engendre systématiquement des proclitiques, met en jeu des contraintes phonologiques, syntaxiques et lexicales ; elle est obligatoire dans certain contextes morphophonologiques et syntaxiques. En créole haïtien, les réductions sont toutes 
facultatives et donnent lieu à des enclitiques et des proclitiques selon les contextes phonologiques décrits par Caddely (1995, 1997) et Valdman (2015). Au niveau syntaxique, ce phénomène met en jeu des élements qui n’entretiennent pas systématiquement de relation de dépendance avec le pronom. Les pronoms objets diffèrent aussi des clitiques objets du français de par leur distribution syntaxique, y compris leur position post-verbale, le fait qu'ils peuvent être coordonnés à un SN et accompagnés de préposition qui indiquent leurs valeurs casuelles et l'agrammaticalité du redoublement de pronom. En ce sens leur comportement n'est pas sans rappeler celui des pronoms objets forts du français (et les pronoms objets de l'anglais). Cette étude confirme donc les analyses du créole haïtien qui traitent les pronoms sujets (Baptista, 1995 ; Cadely, 1995, 1997 ; Déprez, 1994) et objets (Guijarro-Fuente \& Ortiz-Lopez, 2008) comme des clitiques phonologiques, mais des pronoms forts au niveau syntaxique, analyses dorénavant mises à l'épreuve de l'analyse d'échantillons de parole spontanée courante. En ce qui concerne les cooccurrences de pronoms sujets et objets et SN coréférents en créole haïtien, des analyses expérimentales avec des stimuli dont la qualité acoustique et les pauses sont controlées comme dans celles effectuées par Culbertson (2010) pour le registre spontané du français nous permettraient d'en savoir plus sur leurs propriétés distinctives. De plus, des analyses quantitative des contextes d'occurrences des réductions phonologiques nous permettraient aussi de mettre à l'épreuve les principes définis par Cadely $(1995,1997)$ et de dégager un profil aussi précis pour le créole haïtien que celui fourni par les études de Culbertson (2010), Fonseca-Greber et Waugh (2002), Miller (1991) et Morin (1979) pour le français parlé contemporain.

\section{Références bibliographiques}

Armstrong, N. (2002) Variable deletion of French ne: A cross-stylistic perspective. Language Sciences 24.153-73.

Ashby, W. J. (1981) The Loss of the negative particle ne in French: A Syntactic Change in Progress. Language 57.647-687.

Auger, J. (1995). Pronominal Clitics in Québec Colloquial French : A Morphological Analysis. Ph.D. Dissertation, University of Pennsylvania.

Auger, J. (2003). Le redoublement des sujets en picard. French Language Studies (13), 381-406.

Baptista, M. (1995). On the nature of pro-drop in Caperverdean Creole. Harvard Working Papers in Linguistics, 5, 318.

Barrière, I., (2015) New York Haitian Creole Corpus. Unpublished.

Barrière, I., \& Monéreau-Merry, M.M. (2012). Trilingualism of the Haitian Diaspora in NYC: Current et Future Challenges. In O. Garcia, B. Otcu \& Z. Zakharia (Eds.), Bilingual Community Education et Multilingualism: Beyond Heritage Languages in a Global City (pp. 247-258). Bristol, UK: Multilingual Matters.

Bassano, D. ((1998) L’élaboration du lexiqur précoce chez l'enfant français : structure et varabilité. Enfance (4) 123153.

Blanche-Benveniste, C. \& Jeanjean, C. (1987) Le français parlé : transcription et édition. INALF Didier Edition.

Blanche-Benveniste, C., Deulofeu, J., Stéfanini, J. et ven den Eynde, K. (1984) Pronom et syntaxe : l'approche pronominale et son application au français. Paris : SELAF.

Blasco-Dulbecco, M. \& Caddeo, S. (2002) Détachement et linéarité. Recherches sur le français parlé, 17, 41-54.

Cadely, J. J. (1995). Elusion et Agglutination en Créole Haïtien: Le cas des pronoms Personnels. Etudes Créoles, 18(1), 9-38. 
Cadely, J. J. (1997). Prosodie et clicitization en Créole Haïtien: Le métissage des Langues et des représentations. Etudes Créoles, 20(1), 5-114.

Coveney, A. ( 2002) Variability in Spoken French: A sociolinguistic study of interrogation et negation. Exeter: Elm Bank Publications.

Culbertson, J. (2010). Convergent evidence for categorical change in French: from sujet clitic to agreement marker. Language 86 (1), 85-132.

Culbertson, J. \& G. Legendre (2008) Qu'en est-il des clitiques sujet en français oral contemporain? In J. Duret, B. Habert, \& B. Laks (Eds.), Actes du 1er Congrès Mondial de Linguistique Française. Paris : ESN Sciences, 2651-2662.

De Cat, C. (2007) French dislocation: Interpretation, syntax, acquisition. Oxford: Oxford University Press.

Demuth, K. \& Tremblay, A. (2008) Prosodically-conditioned variability in children's production of French determiners. Journal of Child Language 35.99-127.

Detey, S., Duret, J., Laks, B. \& Lyche, C. (éds.) (2010). Les variétés du français parlé dans l'espace francophone: ressources pour l'enseignement (avec DVD). Paris: Ophrys.

Déprez, V. (1994). Haitian Creole: A Pro-Drop Language? Journal of Pidgin et Creole Languages, 9(1), 1-24.

DeGraff, M. F. (1993). Is Haitian Creole a Pro-Drop Language? The Atlantic meet Pacific: A global view of Pidginization et Creolization, 11.

Doetjes, J., Delais-Roussarie, E., \& Sleeman, P. (2002). The Prosody of Left Detached Constituents in French. Proceedings of the Speech Prosody 2002 conference, Aix-en-Provence. 247-250.

Durand, J., Laks, B. \& Lyche, C. (2002) La phonologie du français contemporain: usages, variétés et structure. Romance Corpus Linguistics - Corpora et Spoken Language, ed. by C. Pusch et W. Raible, 93-106. Tübingen: Gunter Narr Verlag.

Durand, J., Laks, B. \& Lyche, C. (2005) La linguistique de corpus, ed. by G. Williams, 205-217. Rennes: Presses Universitaires de Rennes.

Fonseca-Greber, B. \& Waugh, L. (2002) The sujet clitics of Conversational European French: Morphologization, grammatical change, semantic change, et change in progress. A romance perspective on language knowledge et use, ed. by Rafael Núñez-Cedeño, Luis López et Richard Cameron, 99-117. Philadelphia, PA: John Benjamins.

Fuß, E. (2005). The Rise of Agreement. A Formal Approach to the Syntax et Grammaticalization of Verbal Inflection. Amsterdam: J. Benjamins.

Guijarro-Fuente, P. \& Ortiz López, L.A. (2008) Creole/Spanish contact et the Acquisition of Clitics on the Dominican-Haitian Border. International Journal of Bilingualism, 12 (4), 231-262.

Hopper, P. \& Traugott, E. (1993) Grammaticalization. Cambridge: Cambridge University Press.

Kayne, R.S. (1977) Syntaxe du français: le cycle transformationnel. (traduit de l'américain par Pierre Attal). Paris: Seuil. First edition (1975): French Syntax: the transformational cycle. Cambridge, Ms: MIT Press.

Jakubowicz, C. \& Rigaut, C. (1997) L’acquisition des clitiques nominatifs en français. In A. Zribi-Hertz, A. (Ed.) Les pronoms : Morphologie, Syntaxe et Typologie. Vincennes, France : Presses Universitaires.57-99.

Labov, W. (1966) The Social Stratification of English in New York City. Washington: Center for Applied Linguistics. 
Legendre, G., Culbertson, J. ,Barriere, I., Nazzi, T. \& Goyet, L. (2010) Experimental et empirical evidence for the statut et acquisition of sujet clitics et agreement marking in adult et child Spoken French. In Torrens, V., Escobar, L., Gavarro, A., J. Gutierrez (Eds.) Movement et Clitics: Adult et Child Grammar. Newcastle: Cambridge Scholars Publishing. 333-360.

Marandin, J.-M. (2004) Formatage de l'information: focus et contexte. Interpréter en contexte, ed. by Francis Corblin et Claire Gardent, 31-80. Paris: Hermes.

Miller, P. (1991) Clitics et constituents in Phrase Structure Grammar. New York, NY: Garlet.

Morin, Y.C. (1979) La morphophonologie des pronoms clitiques en français populaire. Cahiers de linquistique 9.136.

Pierce, A. (1992) Language acquisition et syntactic theory : a comparative analysis of French et English grammars. Dordrecht: Kluwer.

Plénat, M. (1986) Lexique et phonologie: Observations sur la liaison, la nasalisation, et le comportement des liquides en français standard. Lexique et traitement automatique des langages, 163-185. Toulouse: Université P. Sabatier.

Rizzi, L. (1986) Null objets in Italian et the theory of pro. Linguistic Inquiry 17.501-557.

Ruwet, N. (1976) Problems in French syntax: transformational-Generative Studies (translated by S.M. Robins). London: Longman. Originally published in French (1972) Théorie Syntaxique et Syntaxe du Français. Paris: Seuil.

Samek-Lodovici, V. (2005) Prosody-syntax interaction in the expression of focus. Natural Language et Linguistic Theory 23. 687-755.

Saint Martin, W. (2005) Les Formes des Pronoms Personnels de l'Haïtien et leur place en comparaison avec celles du français. Unpublished Master Thesis, University of Georgia.

Sportiche, D. (1992) Clitic constructions. Phrase structure et the lexicon, ed. by L. Zaring et J. Rooryck, 213-276. Dordrecht: Kluwer Academic Publishers.

Totereau, C., Thevenin, M.G., Fayol, M.(1997) Acquisition de ka morphologie du nombre à l'écrit en français. L. Rieben, M. Fayol \& C.A. Perfetti (dirs.) Des orthographies et leur acquisition. 147-166. Paris: Delachaux et Niestlé.

Valdman, A. (2015) Haitian Creole: Structure, Variation, Status, Origin. Bristol, Connecticut: Equinox.

Valian, V. (1991). Syntactic sujets in the early speech of American et Italian children. Cognition, 40, 21-81.

Zribi-Hertz, A. (1997) Les pronoms : Morphologie, Syntaxe et Typologie. Vincennes, France : Presses Universitaires.

Zwicky, Arnold, et Geoffrey Pullum. 1983. Cliticization vs. inflection: English n’t. Language 59.502-513.

\footnotetext{
${ }^{1}$ Cette étude a été subventionnée par deux bourses de recherche (\#1548147 et 1251707) attribuées à I. Barrière er G. Legendre. Nous remercions les créolophones natifs qui ont participé à notre étude.

${ }^{2}$ L'utilisation des lettres majuscules indique qu'un élément porte l'accent tonique.

${ }^{3}$ Le pronom sujet fort et le pronom sujet clitique féminin de la troisième Personne singulier sont homophones-elletandis que l'équivalent masculin a deux formes distinctes : lui/pronom fort et il/ pronom faible/clitique. Voir le tableau 1 qui présente le paradigme des pronoms sujet et objets forts et faibes/clitiques en français.

${ }^{4}$ Le tableau 2 reflète la variété standard du créole haïtien, variété sur laquelle notre étude porte. La variation dialectale qui affecte la réalisation du pronom sujet de la troisième personne du singulier est l'objet d'une étude sociolinguistique approfondie de Valdman (2015).
} 